\title{
Muller Laurent, Les résidents étrangers à Strasbourg
}

\section{Maurice Blanc}

\section{OpenEdition}

\section{Journals}

Édition électronique

URL : https://journals.openedition.org/remi/6558

DOI : $10.4000 /$ remi.6558

ISSN : $1777-5418$

Éditeur

Université de Poitiers

Édition imprimée

Date de publication : 1 septembre 2013

Pagination : 163-164

ISBN : 979-10-90426-09-2

ISSN : 0765-0752

Référence électronique

Maurice Blanc, "Muller Laurent, Les résidents étrangers à Strasbourg 》, Revue européenne des migrations internationales [En ligne], vol. 29 - n³ | 2013, mis en ligne le 01 septembre 2013, consulté le 15 avril 2022. URL : http://journals.openedition.org/remi/6558; DOI : https://doi.org/10.4000/remi.6558

Ce document a été généré automatiquement le 15 avril 2022.

(C) Université de Poitiers 


\title{
Muller Laurent, Les résidents étrangers à Strasbourg
}

\author{
Maurice Blanc
}

\section{RÉFÉRENCE}

Muller Laurent (2009) Les résidents étrangers à Strasbourg, Strasbourg, Presses universitaires de Strasbourg, 288 p., ISBN : 978-2-86820-380-9.

1 Laurent Muller est maître de conférences à l'université de Strasbourg et il a intitulé cet ouvrage : Les résidents étrangers à Strasbourg. Ce titre est vrai et faux à la fois : il est bien question des étrangers à Strasbourg, mais ce n'est pas une étude monographique de plus. En reprenant une expression de Michel Marié (Les Terres et les Mots, Klincksieck, 1989), l'étranger a une "fonction-miroir»: il est le révélateur de la société strasbourgeoise. Quand les Strasbourgeois parlent de «leurs » étrangers, leur discours nous apprend autant (sinon plus) sur les Strasbourgeois eux-mêmes que sur les étrangers. Les étrangers servent aussi de prétexte à un projet scientifique ambitieux, que l'auteur dévoile par petites touches successives (voir infra).

2 Strasbourg a été ballotée et tiraillée entre la France et l'Allemagne. Elle est à la fois une capitale régionale et une des capitales de l'Europe. Elle affirme en bloc son attachement à la France et à ses propres spécificités linguistiques, culturelles et même juridiques (le droit local). C'est un bon «observatoire social» sur le rapport à l'étranger, qui entremêle la proximité et la distance; mais Strasbourg n'est pas une exception pour autant. Sous des déclinaisons spécifiques, les dynamiques sociales analysées ici sont aussi à l'œuvre dans les villes d'Europe et d'ailleurs.

3 Laurent Muller combine judicieusement les données statistiques au niveau des quartiers avec les travaux historiques, l'analyse de contenu de la presse régionale et nationale, l'observation ethnographique dans les transports en commun ou au domicile des enquêtés, lorsqu'il réalise des entretiens biographiques. Il utilise les travaux des étudiants qu'il forme à la recherche et par la recherche, les associant à l'analyse et à la 
publication des résultats. Mais il a le défaut d'écrire pour des lecteurs strasbourgeois, sans tenir suffisamment compte des autres. Entre autres exemples qui, en dehors de Strasbourg, connaît le " quartier des Quinze » et qui se souvient encore du " tandem » Fabienne Keller et Robert Grossmann, aux commandes de la ville de 2001 à 2008, tous les deux dans la majorité présidentielle de l'époque (la première maire de la Ville, le second président de la Communauté urbaine) ? Sans ces connaissances élémentaires, il est difficile de comprendre les analyses de la politique locale.

Laurent Muller s'inspire de deux sociologues qui ont vécu un temps à Strasbourg: Georg Simmel, précurseur de la sociologie urbaine et auteur entre autres des Digressions sur l'étranger, a fini sa vie et sa carrière universitaire en 1918 à Strasbourg, alors allemande ; Maurice Halbwachs, auteur des Cadres sociaux de la mémoire, a été fusillé par les Allemands pendant la Seconde Guerre mondiale. L'auteur les associe en considérant qu'il y a cinq figures (ou formes sociales de visibilité) de l'étranger et qu'elles constituent autant de " cadres sociaux de la mémoire ».

5 (1) La visibilité de l'étranger est d'abord évolutive, car elle est prise dans un couple attraction/répulsion, oscillant d'un processus à l'autre, ou les faisant cohabiter.

(2) La visibilité différentielle, entre les étrangers « chics » dans le quartier des diplomates et les étrangers "chocs» dans les grands ensembles de logements sociaux des banlieues. De ce point de vue ce sont les transports urbains et les commerces qui sont des lieux privilégiés pour étudier la mobilité et l'échange social.

(3) La visibilité concédée se manifeste notamment lors de l'expérience du Conseil consultatif des étrangers, menée par la maire socialiste de la ville, Catherine Trautmann. Projet phare d'une timide politique de «discrimination positive», elle a provoqué de vives réactions de rejet, à la fois par le Front National et par l'éphémère Parti des Musulmans de France, créé à Strasbourg.

(4) La visibilité conquise est d'abord le fruit de la dynamique des associations d'étrangers et des associations de solidarité qui les soutiennent; les rencontres interculturelles, émanant souvent des centres socio-culturels dans les quartiers, et la mise en valeur de l'histoire et de la mémoire de l'immigration, contribuent aussi à cette conquête de la visibilité. La presse locale adopte alors un ton plus mesuré pour parler de l'islam, des réfugiés ou des sans-papiers, etc.

(5) La visibilité négociée, se situe au niveau anthropologique de la famille et de la vie quotidienne: négociations entre hommes et femmes, parents et enfants, avec les voisins, etc.

(6) Sans la développer, l'auteur suggère une sixième figure, l'étranger suppliant, en reprenant une formule du philosophe Paul Ricœur.

6 En fin de la première partie, l'auteur écrit: «la visibilité évolutive de l'étranger se dispute dans l'imaginaire collectif alsacien entre l'attrait pour l'exotisme et la peur de l'inconnu » (p. 66), ce qui n'a rien de spécifiquement alsacien. De même, page 266 le titre «Strasbourg comme cadre social total » suggère que Strasbourg a des formes qui lui sont propres, certes, mais est-elle une exception? Peut-être en raison d'un scrupule scientifique excessif, l'auteur s'interdit toute montée en généralité et reste dans "sa » ville. On aurait souhaité, au moins à titre d'hypothèse à vérifier par des recherches futures, que toute ville soit un cadre social total.

7 La conclusion générale fait une large place à la vie et l'œuvre de Simmel, ce qui aurait mérité de venir dès l'introduction. Mais c'est peut-être le véritable objet de cet ouvrage qui est ainsi dévoilé. La faculté des sciences sociales de Strasbourg a une longue 
tradition d'études simméliennes et elle contribue à diffuser sa pensée en France, participant ainsi au renouveau de la sociologie urbaine et, plus largement, de toute la sociologie en France ${ }^{1}$. Laurent Muller s'inscrit dans cette tradition et il en montre la fécondité.

\section{NOTES}

1. Le numéro spécial de la Revue des Sciences Sociales, Strasbourg, carrefour des sociologies, 40 (2008), a consacré plusieurs contributions à Simmel et Halbwachs. Il fait de Strasbourg un point de rencontre des sociologies française et allemande, donc un lieu d'hybridation, de métissage et d'innovations sociologiques.

\section{AUTEURS}

\section{MAURICE BLANC}

Sociologue, professeur émérite, SAGE (Sociétés, Acteurs, Gouvernements, Europe), CNRS/

Université de Strasbourg 